\title{
The detection of reverse accumulation effect in the positron annihilation profile of stack of aluminum and silver foils
}

\begin{abstract}
The implantation profile of positrons emitted from ${ }^{22} \mathrm{Na}$ into a stack of aluminum and silver foils is the subject of the presented report. The characteristic dimple in the profile behind the Ag foil was detected. This effect arises from the differences in the linear absorption coefficient of aluminum and silver. The good agreement between the theoretical profile obtained within the multiscattering model and experimental one was achieved. The observed phenomenon can affect the positron annihilation characteristics measured for the inhomogeneous samples.
\end{abstract}

Key words: positron implantation profile $\bullet$ stack of foils

J. Dryzek ${ }^{\bowtie}$ K. Siemek

Institute of Nuclear Physics of the Polish Academy of Sciences,

152 Radzikowskiego Str., 31-342 Kraków, Poland, Tel.: +48 12662 8438, Fax: +48 12662 8458,

E-mail: jerzy.dryzek@ifj.edu.pl

Received: 18 June 2015

Accepted: 13 August 2015

\section{Introduction}

The transport of energetic positrons (or electrons) in matter is accompanied by several phenomena. Generally, this can be divided into three stages, implantation, thermalization, and diffusion, and finally, annihilation with electrons. During implantation, positron penetrates the largest volume in the shortest time; however, during thermalization and diffusion, positron spends the largest time scanning and much smaller volume. On close inspection, definite boundaries between the three processes cannot be determined. For spectroscopy, the most important is the diffusion process. In this case, scattering with phonons dominates, and finally, annihilation with electrons takes place. However, implantation process determines the range. In this case, we can numerate the following processes: elastically scatter without loss of energy, emission of braking photons while scattering from the nucleus (Bremsstrahlung effect), inelastically scatter by raising an atom to the excited state and kick out one of an atomic electron and ionize the atom [1]. This electron can have an appreciable range of its own is called as the $\delta$-ray. The loss of energy by positrons is involved by Bremsstrahlung and the excitation and ionization. We should note that annihilation of fast positrons called as annihilation in flight is also possible, but the cross section for this process is very small. For each of these processes, a cross section strictly depends on the type of the atom; thus implantation into inhomogeneous medium, which contain several type of atoms or their precipitation, can complicate the transport phenomena significantly. 
During implantation, positrons lose their energy. The occurrence of very frequent, small energy losses along the path leads to the concept of stopping power, which is defined as the average energy loss, $d E$, per unit distance $d s$ along the track. This is suitable for the monoenergetic beam of positrons. Another concept for the description of the implantation process is the linear absorption coefficient whose reciprocal value is the average implantation depth. This is convenient parameter for the positrons or electrons emitted from the radioactive sources in the $\beta^{+}$decay. Both values strictly depend on the atomic number and atomic mass of implanted medium because Coulomb interaction is the main interaction of charged particles.

In our recent studies, we noticed that passage of energetic positrons through a stack of different metal foils results in the characteristic increase in the number of positrons annihilating in denser foil surrounded by less dense foils [2]. This effect can be simply explained if only the linear absorption coefficient is taken into account. This is phenomenological parameter which in many application is sufficient to characterize spatial distribution of implanted from radioactive sources positrons. Its value depends on the end point of energetic positron spectra, density of the implanted medium. Thus denser regions absorb more positrons than less dense. This effect, called by us as positron accumulation effect, was also experimentally detected for 3D precipitates embedded in a low-density matrix [3]. However, the reverse accumulation effect is also expected. In the paper, we intend to present the experimental results that confirm it.

\section{The accumulation and reverse accumulation effect}

Let us consider the modeled stack of silver foil embedded between two aluminum foils, each foils is $10 \mu \mathrm{m}$ thick, Fig. 1 . The ${ }^{22} \mathrm{Na}$ positrons are implanted into the stack. Assuming the normalized number of transmitted positrons at the depth $z$ from the source is described by the exponential decay function, then this function for the stack is as follows:

(1) $I(z)=\left\{\begin{array}{lr}\exp \left(-\alpha_{\mathrm{Al}} z\right), & z<10 \mu \mathrm{m} \\ \exp \left[-\left(\alpha_{\mathrm{Al}}-\alpha_{\mathrm{Ag}}\right) d_{\mathrm{Ag}}\right] \exp \left(-\alpha_{\mathrm{Ag}} z\right), & 10 \mu \mathrm{m} \leq z \leq 20 \mu \mathrm{m} \\ \exp \left[-\left(\alpha_{\mathrm{Ag}}-\alpha_{\mathrm{Al}}\right) d_{\mathrm{Ag}}\right] \exp \left(-\alpha_{\mathrm{Al}} z\right), & z>20 \mu \mathrm{m}\end{array}\right.$

where linear absorption coefficient in aluminum and silver is equal to $\alpha_{\mathrm{Al}}=114$ and $\alpha_{\mathrm{Ag}}=554 \mathrm{~cm}^{-1}$, respectively, and $d_{\mathrm{Ag}}=10 \mathrm{~mm}$. The top solid line in Fig. 1 represents this function. The positron implantation profile represents the normalized number of positrons that annihilate at the depth between $z$ and $z+d z$ from the source given as follows:

$$
P(z)=-\frac{d I(z)}{d z}
$$

while the transmittance function is continuous, the implantation profile, driven in the bottom line in Fig. 1, exhibits discontinuous at the interfaces. In silver foil, positrons are accumulated, and we call

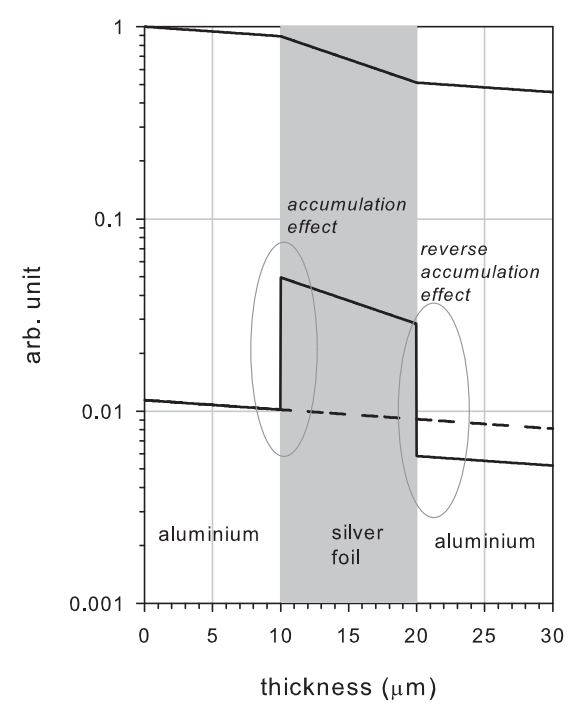

Fig. 1. The comparison of the experimental positron implantation profile obtained using DSIP measurement (gray circles) and calculated using LYS-1 program for stacks of plates and foils, which scheme depicted in the top is as follows: $\mathrm{Al}(2 \mathrm{~mm})-\mathrm{Ag}(40 \mu \mathrm{m})-\mathrm{Al}(20 \mu \mathrm{m})-{ }^{22} \mathrm{Na}-\mathrm{Al}$ $(2 \mathrm{~mm})$. The solid line represents the calculated profile and the solid curve the profile after convolution.

it as the accumulation effect. However, we have also second discontinuous at the depth of $20 \mu \mathrm{m}$ when positrons passage from silver to aluminum foil. In this case, it looks that the number of positrons is relatively diminish, then we call it as the reverse accumulation effect (r-accumulation effect). The comparison with the profile where instead of silver aluminum foil is given in Fig. 1 as the dashed line. Thus in the implantation profile, one should observe a dimple for reverse and a bump for accumulation effect. The bump can be detected experimentally. For the detection of a dimple, we apply the depth scanning of the implantation profile (DSIP) method.

\section{The experimental details}

The experimental technique called DSIP is dedicated for the detection of positron implantation profiles in different samples [4]. In this experiment, we applied the high-purity germanium (HPGe) detector for the detection of annihilation photons emitted only from selected regions of the sample where positrons were implanted. For selecting the region of a sample, we constructed the collimator with a very narrow slit with the width of $60 \mu \mathrm{m}$ behind which the detector was located. By moving the sample with the positron emitter perpendicularly in front of the collimator and measuring the area under the annihilation peak, we obtained the information on the positron profile in the studied sample. This technique is described in detail in our previous paper [4]. In order to improve the background reduction and observe more details in the profile, we decided also to record the second annihilation photon. The HPGe detector is placed behind a $10-\mathrm{cm}$-thick shield made from a tungsten-based heavy metal (TRIAMET with 95\% tungsten) with a long slit of $60 \mu \mathrm{m}$ in width. The 
second detector with $\mathrm{NaI}(\mathrm{Tl})$ scintillator is located behind another collimator made of 10 -cm-thick lead shield with a long slit of $1 \mathrm{~mm}$ in width. This detector is used only for monitoring the $511 \mathrm{keV}$ annihilation photon. If it appears, the signal opens the gate for the signals from the HPGe detector, which are collected in the multichannel analyzer. This coincidence mode reduces the counting rate by the factor of about 100 in comparison to the mode when signals from the HPGe are not gated. In our experiment, the ${ }^{22} \mathrm{Na}$ radioactive source of $25 \mu \mathrm{Ci}$ activity as a positron emitter was sandwiched between two samples.

\section{The experimental results and discussion}

For searching the r-accumulation effect, we constructed a stack that consists of the aluminum plate of $2 \mathrm{~mm}$ thick, the ${ }^{22} \mathrm{Na}$ positron source enveloped in the 7-mm-thick kapton foil, the aluminum foil of $20 \mu \mathrm{m}$ thick, and the silver foil of $40 \mu \mathrm{m}$ thick. The stack was pressed by 2 -mm-thick aluminum plate. Our interest is focused on the profile of energetic positrons; thus their random walk with thermal energy can be neglected. This is well justified, the typical diffusion length of thermalized positrons is of order of $0.1 \mu \mathrm{m}$, the typical implantation depth is about a few dozens or hundreds of micrometers, and for comparison, the thickness of the foils used in our experiment is at least $20 \mu \mathrm{m}$. Additionally, the foils in the stack were not sealed. The schema of the stack is presented in the top of Fig. 2. The circles in Fig. 2 represent the measured implantation profile using DSIP method. On the right side from the ${ }^{22} \mathrm{Na}$ source, only the aluminum plate is present and the profile decay gradually; it describes the exponential decay function with the good approximation. On the left side, the profile also decay but the characteristic dimple behind the Ag foil is clearly visible.

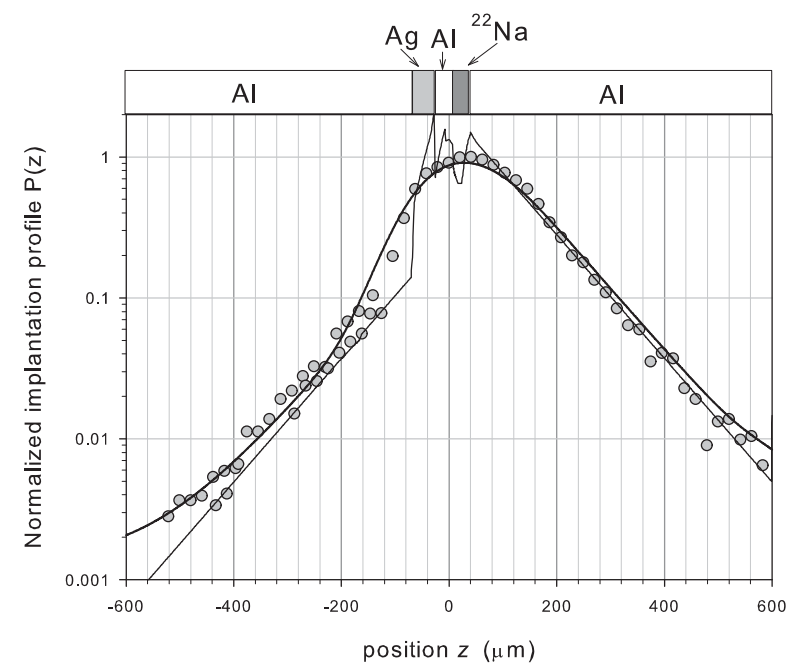

Fig. 2. The modeled schema of the stack of $\mathrm{Al}(10 \mu \mathrm{m})-$ $\mathrm{Ag}(10 \mu \mathrm{m})-\mathrm{Al}(10 \mu \mathrm{m})$ for illustration of the accumulation and r-accumulation effects. The ${ }^{22} \mathrm{Na}$ positrons are implanted from the left side. The top solid line represents the transmittance function, Eq. (1), and the solid bottom line represents the positron implantation profile, Eq. (2). The dashed line is the profile for the case when the silver foil is replaced by the aluminum foil of the same thickness.
This is the searched r-accumulation effect; to proof it, the calculations using the multiscattering model were performed. For describing the implantation profile in this model, only absorption and backscattering at interfaces between foils are taken into account [2]. The mathematical formulas of the model were implemented into the LYS-1 (LaYerS) program, which is available online [5]. The use of this code allowed us to generate the implantation profile which is represented in Fig. 2 as the solid line. The line contains several twists, which are direct at interfaces, between source and plate, and between $\mathrm{Al}$ and $\mathrm{Ag}$ foils. The largest twist between $\mathrm{Al}$ and $\mathrm{Ag}$ foil corresponds to the r-accumulation effect considered above and represented in Fig. 2. The generated profile cannot be compared with the experimental profile because the latter contains also the spatial resolution function. For direct comparison with the experimental profile, the generated implantation profile is convoluted with a Gaussian resolution function with full width at half maximum (FWHM) equal to the width of slit, i.e. $60 \mu \mathrm{m}$. Also the background was added to the profile with ratio $1: 1000$. The convoluted implantation profile is shown in Fig. 2 as a solid curve. The good agreement with the experimental profile is achieved. We should note that nonfitting procedure was applied. Only the phenomenological parameters such as linear absorption and backscattering coefficient were used. The following values of $\alpha_{\mathrm{Al}}=101 \mathrm{~cm}^{-1}, \alpha_{\mathrm{Ag}}=300 \mathrm{~cm}^{-1}$, the backscattering coefficient $R_{\mathrm{Al}}=0.23$ and $R_{\mathrm{Ag}}=$ 0.6 were used to obtain a good agreement with the experimental profile. The lower value of the linear absorption coefficient for sliver foil in comparison to the value obtained from the simple relation reported in literature and equals to $554 \mathrm{~cm}^{-1}$ should be noted. This can be explained because positrons that penetrate silver foil first must passage thought an aluminum foil of $20 \mathrm{~mm}$ thick. Their energetic spectra is modified, and this can be reflected in the value of the linear absorption coefficient.

The presence of the r-accumulation effect should be accompanied by the accumulation effect according to Fig. 1. However, in Fig. 2, no bump is visible. This is due to the poor spatial resolution of the DSIP system. The thickness of the silver foil is $40 \mu \mathrm{m}$ and is less than the width of the slit; thus for this stack, it is difficult to observe accumulation effect but not the r-accumulation because the thickness of the aluminum plate behind the silver foil is much higher. For direct observation of the accumulation effect, another stack should be constructed. In our previous paper, we use, for instance, the stack as follows: aluminum plate of $2 \mathrm{~mm}$ thick $-{ }^{22} \mathrm{Na}$-aluminum foil of $200 \mu \mathrm{m}$ thick - titanium plate of $2 \mathrm{~mm}$ thick [2]. In this case, the bump in the titanium plate region was clearly visible in the implantation profile.

The r-accumulation effect can affect the positron annihilation characteristics measured in the positron spectroscopy for inhomogeneous samples similar to the accumulation effect. Recently, in our studies of samples with particles embedded in a low-density matrix, we have detected that because of the accumulation effect, the fraction of positrons 
that annihilate in the particles excides their volume fraction. This is clearly visible in the samples when tungsten particles are embedded in an epoxy resin. We can reverse this case and consider samples with the low-density particles embedded in high-density matrix. We argue that because of the r-accumulation effect, the fraction of positrons in these particles is lower than their volume fraction.

\section{Conclusions}

The measurements of the positron implantation profile in a stack of aluminum plates, aluminum foils, and silver foil revealed the characteristic dimple behind the silver foil. This effect can be simply explained because of the differences in the linear absorption coefficients of aluminum and sliver. The detail calculations using the multiscattering model allows us to obtain the good agreement between theoretical and measured profile.

\section{References}

1. Carron, N. J. (2007). An introduction to the passage of energetic particles through matter. New York: Taylor and Francis.

2. Dryzek, J., \& Siemek, K. (2013). The accumulation effect of positrons in the stack of foils, detected by measurements of the positron implantation profile. J. Appl. Phys., 114, 224901-224906. DOI: 10.1063/1.4843035.

3. Dryzek, J., \& Siemek, K. (2015). Positron accumulation effect in particles embedded in a low-density matrix. $J$. Appl. Phys., 117, 055901-7. DOI: 10.1063/1.4906400.

4. Dryzek, J. (2005). Defect depth scanning over the positron implantation profile in aluminum. Appl. Phys. A, 81, 1099-1104. DOI: 10.1007/s00339-0042966-6.

5. http://www.ifj.edu.pl/ mdryzek/index.html for LYS-1 program. 